Supporting Information

\title{
Solid-State Organic Electrolyte-Gated Transistors based on Doping- Controlled Polymer Composites with a Confined Two-Dimensional Channel in Dry Conditions
}

Donguk Kim, ${ }^{1}$ Hong Jang, ${ }^{2}$ Seungjin Lee, ${ }^{1}$ Bumjoon J. Kim,,${ }^{1, *}$ and Felix Sunjoo Kim ${ }^{2, *}$

${ }^{1}$ Department of Chemical and Biomolecular Engineering, Korea Advanced Institute of Science and Technology (KAIST), Daejeon 34141, Republic of Korea

${ }^{2}$ School of Chemical Engineering and Materials Science, Chung-Ang University (CAU), Seoul 06974, Republic of Korea

* Corresponding Authors: [B.J.K.] bumjoonkim@kaist.ac.kr; [F.S.K.] fskim@cau.ac.kr 

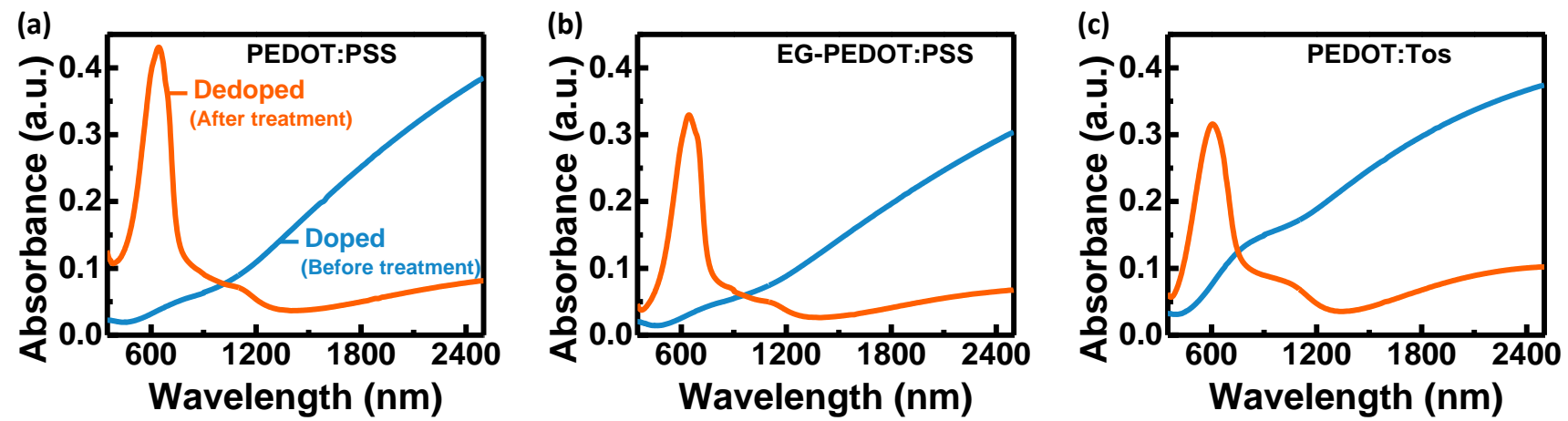

Figure S1. UV-Vis-NIR absorption spectra of various PEDOT composites before and after TDAE treatment: (a) PEDOT:PSS, (b) EG-PEDOT:PSS, and (c) PEDOT:Tos. 

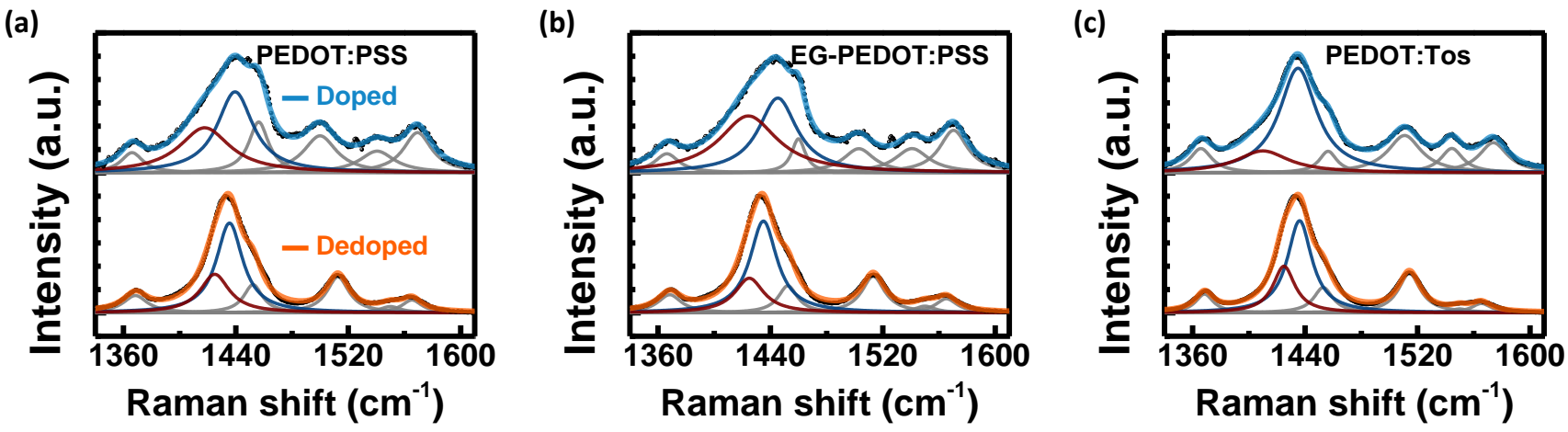

Figure S2. Raman spectra of various PEDOT composites: (a) PEDOT:PSS, (b) EG-PEDOT:PSS, and (c) PEDOT:Tos. The blue and red colored subpeaks are associated with benzoid and quinoid resonant structures, respectively.

Table S1. Deconvoluted peak parameters of Raman scattering for symmetric $\mathrm{C}_{\alpha}=\mathrm{C}_{\beta}$ stretching.

\begin{tabular}{|c|c|c|c|c|c|c|c|}
\hline \multicolumn{2}{|c|}{$\mathrm{C}_{\alpha}=\mathrm{C}_{\beta}$ symmetric stretching } & \multicolumn{2}{|c|}{ PEDOT:PSS } & \multicolumn{2}{|c|}{ EG-PEDOT:PSS } & \multicolumn{2}{|c|}{ PEDOT:Tos } \\
\hline & & Doped & Dedoped & Doped & Dedoped & Doped & Dedoped \\
\hline \multirow{3}{*}{ 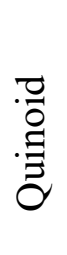 } & Position $\left(\mathrm{cm}^{-1}\right)$ & 1417.9 & 1425.0 & 1424.3 & 1425.0 & 1410.0 & 1425.0 \\
\hline & Peak Area $(\%)$ & 44.1 & 32.2 & 53.6 & 28.7 & 22.7 & 31.3 \\
\hline & FWHM $\left(\mathrm{cm}^{-1}\right)$ & 43.9 & 25.1 & 50.1 & 24.9 & 44.9 & 19.0 \\
\hline \multirow{3}{*}{ 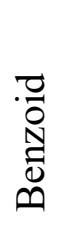 } & Position $\left(\mathrm{cm}^{-1}\right)$ & 1439.5 & 1435.5 & 1445.3 & 1435.0 & 1435.0 & 1436.1 \\
\hline & Peak Area (\%) & 55.9 & 67.8 & 46.4 & 71.3 & 77.3 & 68.7 \\
\hline & FWHM $\left(\mathrm{cm}^{-1}\right)$ & 30.9 & 22.7 & 33.0 & 23.3 & 32.1 & 21.0 \\
\hline
\end{tabular}



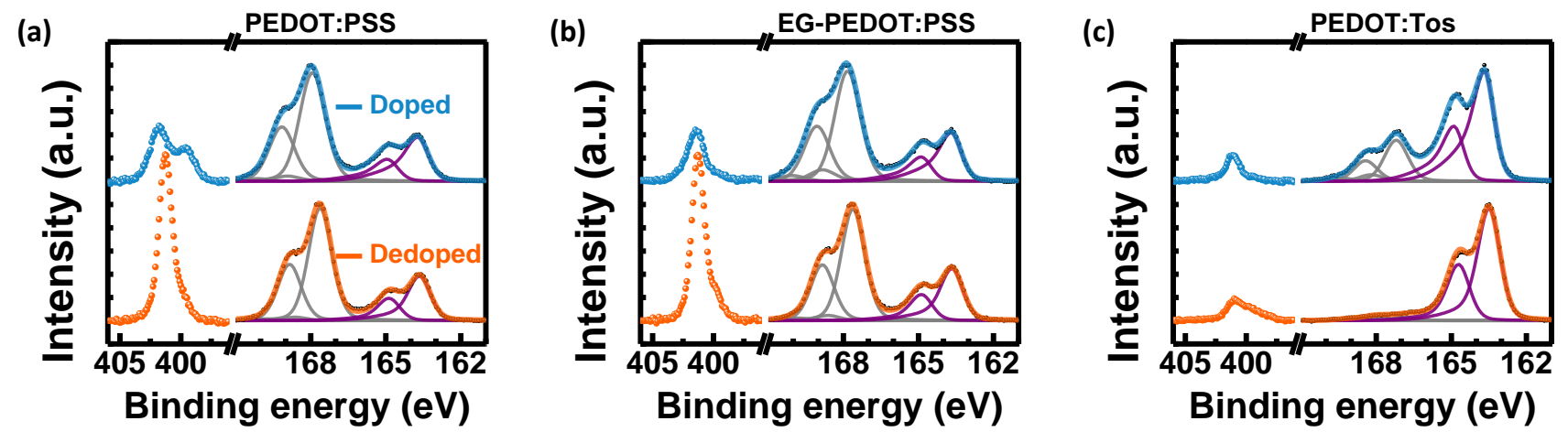

Figure S3. $N$ 1s and S 2p XPS spectra of various PEDOT composites: (a) PEDOT:PSS, (b) EGPEDOT:PSS, and (c) PEDOT:Tos. The violet-colored subpeaks originate from slightly doped PEDOT chains in the PEDOT composites.

Table S2. Calculated weight ratios of sulfonates to EDOT and atomic ratios of $\mathrm{N}$ to EDOT based on peak fitting.

\begin{tabular}{ccccccc}
\hline & \multicolumn{2}{c}{ PEDOT:PSS } & \multicolumn{2}{c}{ EG-PEDOT:PSS } & \multicolumn{2}{c}{ PEDOT:Tos } \\
& Doped & Dedoped & Doped & Dedoped & Doped & Dedoped \\
\hline Sulfonate/EDOT wt. ratio & 2.22 & 2.62 & 2.31 & 2.26 & 0.38 & 0.06 \\
N 1s/EDOT at. ratio & 1.24 & 2.51 & 0.75 & 2.12 & 0.15 & 0.23 \\
\hline
\end{tabular}



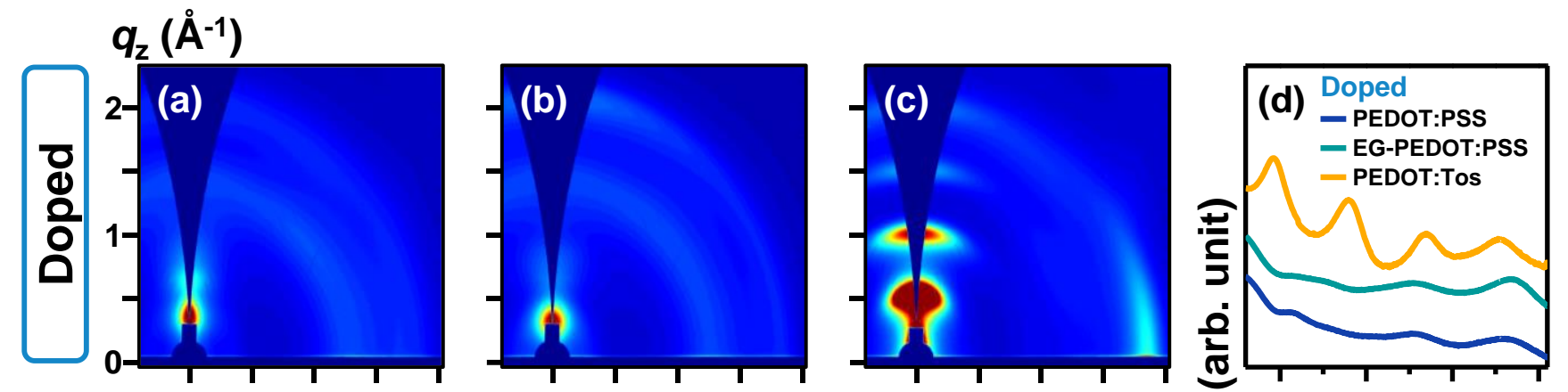

PEDOT:PSS
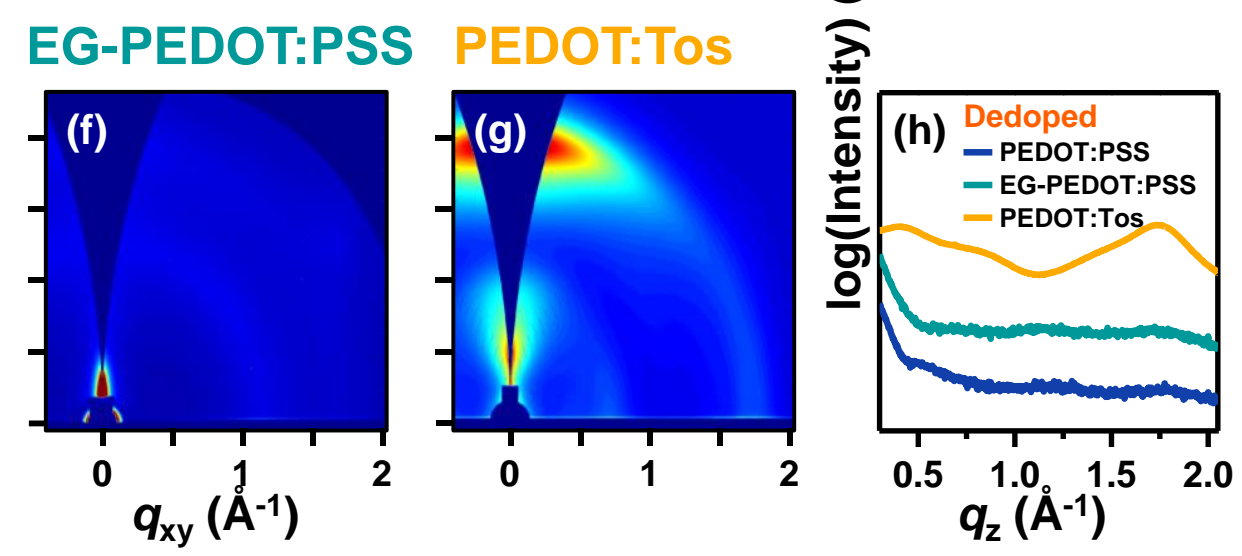

Figure S4. Morphological characterization of various PEDOT composites. 2D GIWAXS pattern and their out-of-plane line-cuts for $(\mathrm{a}-\mathrm{d})$ doped and $(\mathrm{e}-\mathrm{h})$ dedoped PEDOT composite films. 


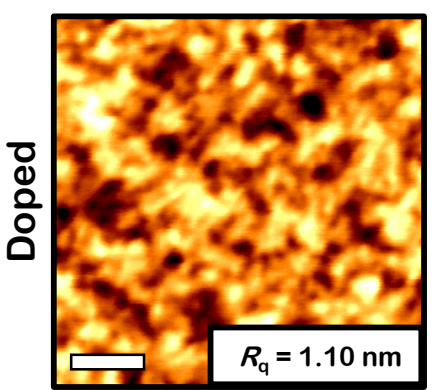

PEDOT:PSS
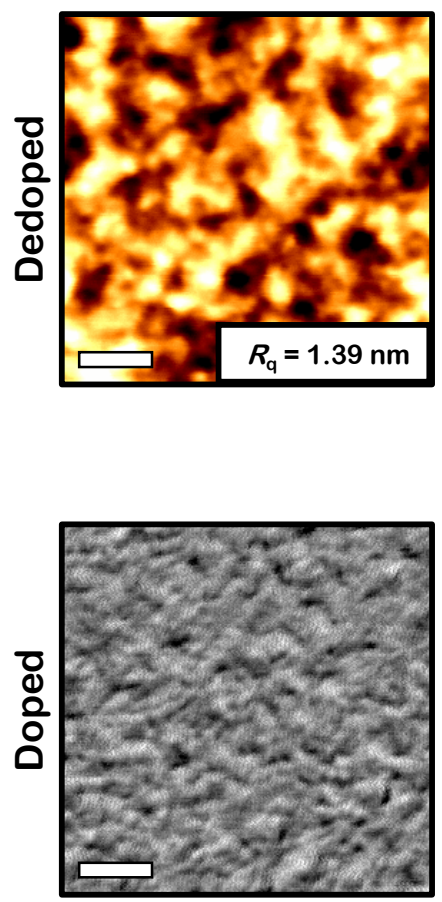

PEDOT:PSS

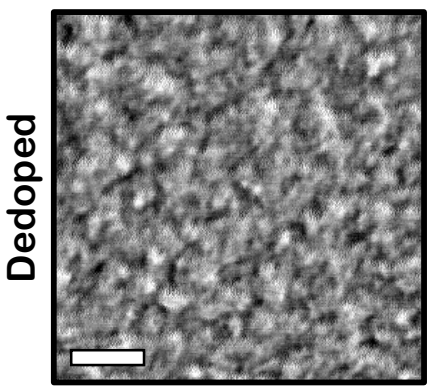

Topography

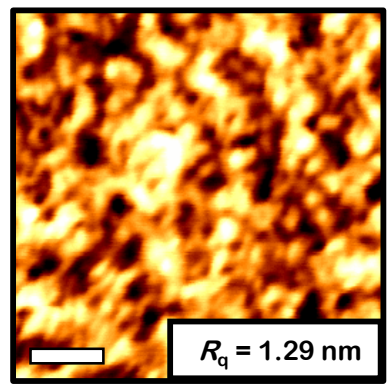

EG-PEDOT:PSS

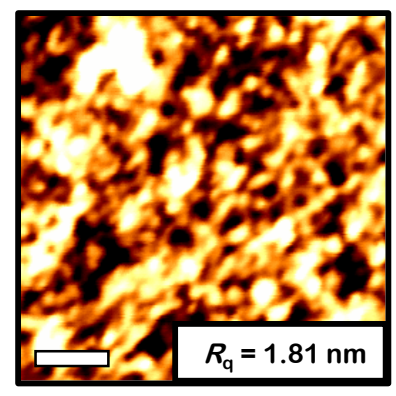

Phase

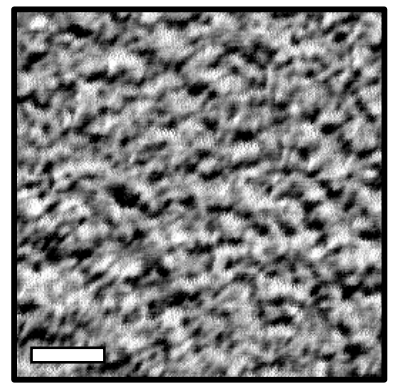

EG-PEDOT:PSS

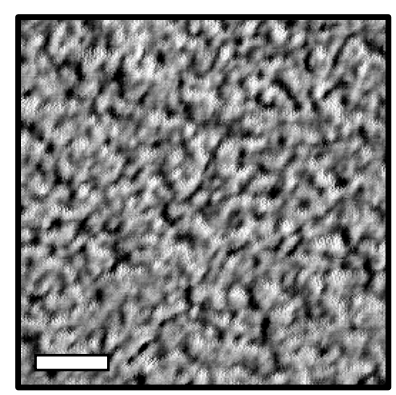

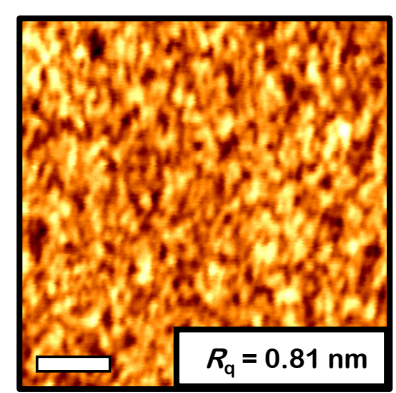

PEDOT:Tos
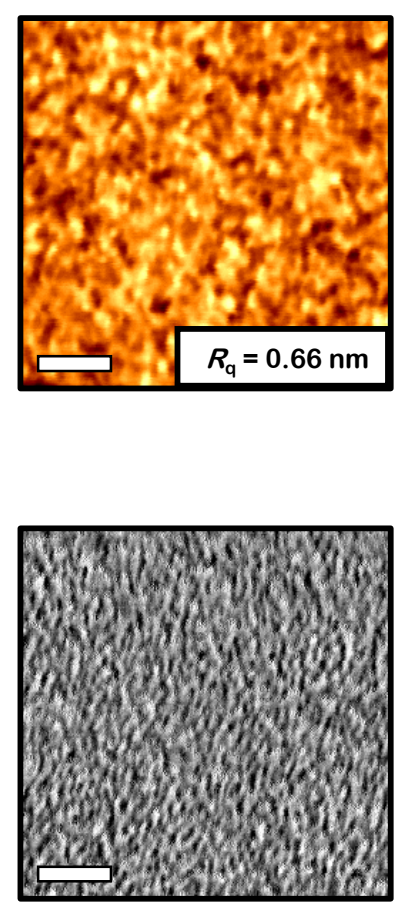

PEDOT:Tos

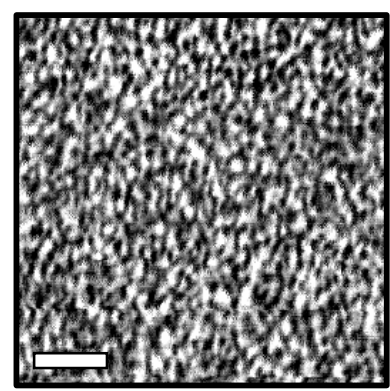

$0 \mathrm{~nm}$

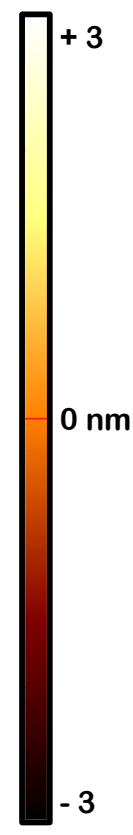

3

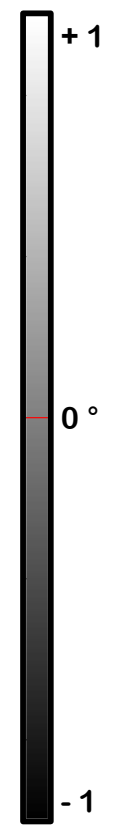

Figure S5. Topography and phase images of PEDOT composite films obtained by atomic force microscopy. The scale bar represents $200 \mathrm{~nm}$. 
(a)

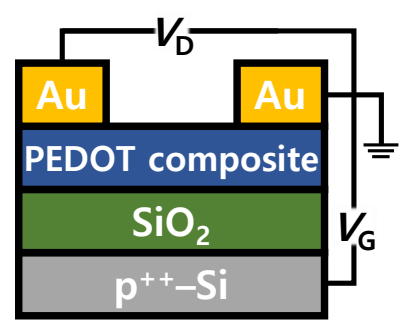

(d)

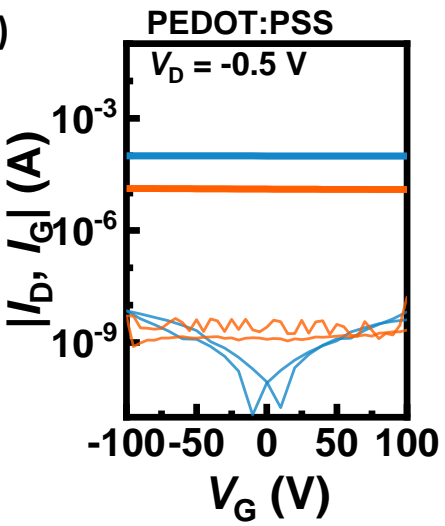

(g)

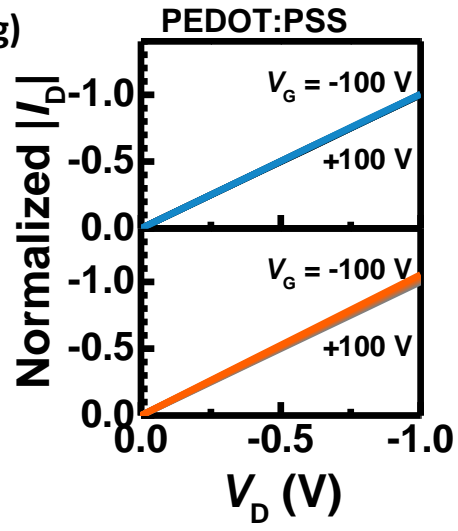

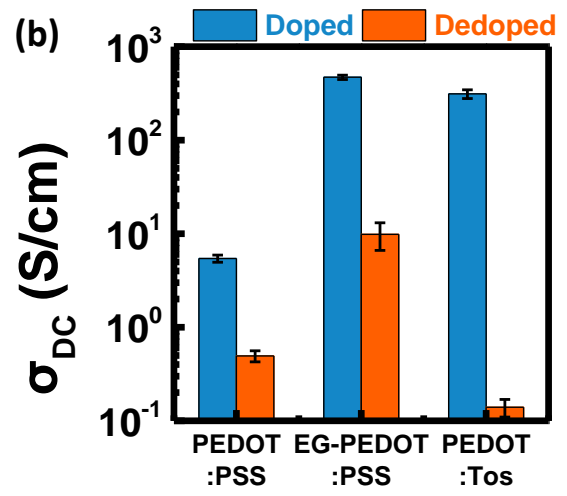

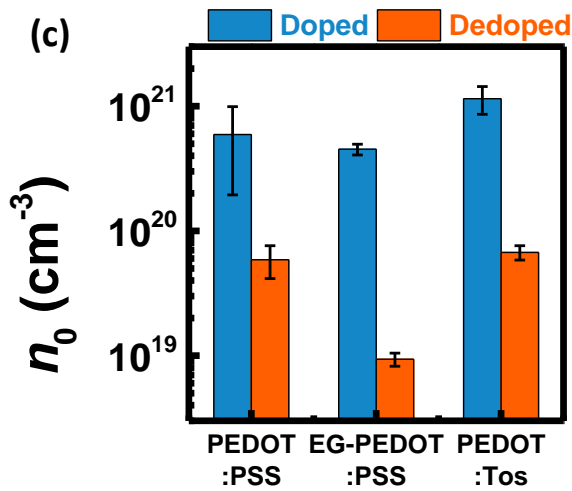

(e)

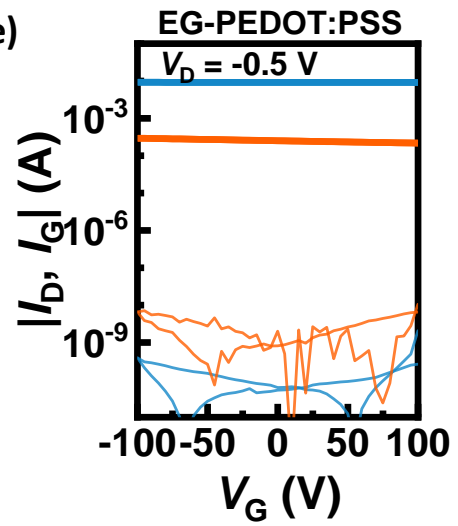

(h)

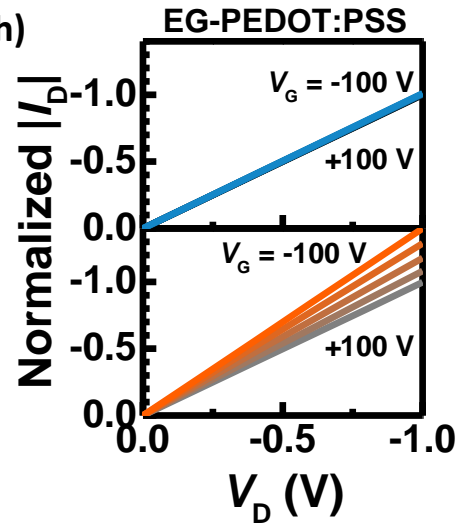

(f)

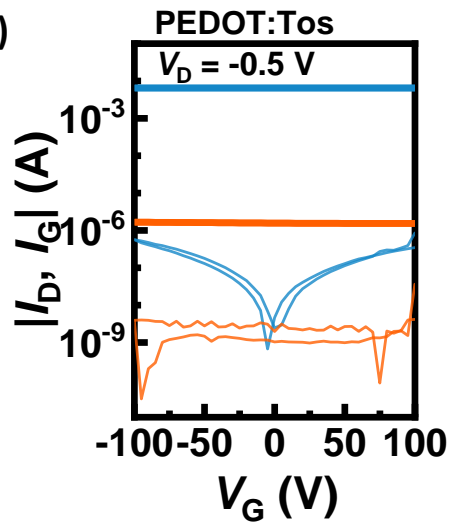

(i)

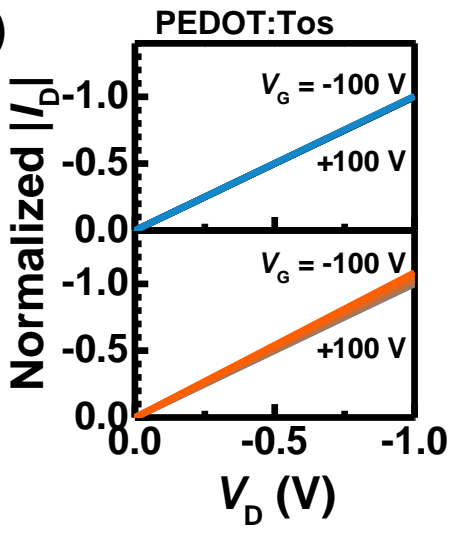

Figure S6. Electrical characteristics of various PEDOT composites and OFET devices: (a) Schematic representation of OFET devices. (b) Thin-film electrical conductivity measured by a four-point-probe method. (c) Charge-carrier density calculated from the conductivity and the linear-regime field-effect mobility. (d-f) Transfer curves and (g-i) output curves from OFETs with low-capacitance $\mathrm{SiO}_{2}(200 \mathrm{~nm})$ dielectric layer. 
Table S3. Electrical parameters from OFETs with a low-capacitance $\mathrm{SiO}_{2}(200 \mathrm{~nm})$ dielectric layer.

\begin{tabular}{ccccccc}
\hline & \multicolumn{2}{c}{ PEDOT:PSS } & \multicolumn{2}{c}{ EG-PEDOT:PSS } & \multicolumn{2}{c}{ PEDOT:Tos } \\
& Doped & Dedoped & Doped & Dedoped & Doped & Dedoped \\
\hline$\sigma_{\mathrm{DC}}^{\mathrm{a}}\left(\mathrm{S} \mathrm{cm}^{-1}\right)$ & 5.43 & 0.49 & 471 & 9.8 & 312 & 0.13 \\
& \pm 0.48 & \pm 0.07 & \pm 26 & \pm 3.2 & \pm 33 & \pm 0.03 \\
$\mu_{\mathrm{Lin}, \mathrm{FET}}{ }^{\mathrm{b}}\left(\mathrm{cm}^{2} \mathrm{~V}^{-1} \mathrm{~s}^{-1}\right)$ & 0.114 & 0.040 & 5.791 & 5.504 & 1.890 & 0.011 \\
$V_{\mathrm{th}}^{\mathrm{c}}(\mathrm{V})$ & \pm 0.062 & \pm 0.019 & \pm 0.176 & \pm 0.997 & \pm 0.430 & \pm 0.004 \\
$I_{\mathrm{ON}} / I_{\mathrm{OFF}}{ }^{\mathrm{d}}$ & 18669 & 3045 & 17869 & 580 & 41589 & 1573 \\
$n_{0, \mathrm{Vth}}=C_{\mathrm{i}} V_{\mathrm{th}} / e d^{\mathrm{e}}\left(\mathrm{cm}^{-3}\right)$ & 1.008 & 1.064 & 1.011 & 1.410 & 1.004 & 1.104 \\
$n_{0, \mathrm{Cond}}=\sigma_{\mathrm{DC}} / e \mu_{\mathrm{Lin}, \mathrm{FET}}{ }^{\mathrm{f}}\left(\mathrm{cm}^{-3}\right)$ & $3.0 \times 10^{20}$ & $7.6 \times 10^{19}$ & $5.1 \times 10^{20}$ & $1.1 \times 10^{19}$ & $1.0 \times 10^{21}$ & $8.2 \times 10^{19}$ \\
\hline
\end{tabular}

a. Thin film conductivity achieved by using the four-point probed measurement.

b. Charge-carrier mobility extracted by linear regime MOSFET equation, $I_{\mathrm{D}}=(W / L)\left(\mu_{\mathrm{Lin}, \mathrm{FET}} C_{\mathrm{i}}\right)\left(V_{\mathrm{G}}-V_{\mathrm{th}}-0.5 V_{\mathrm{D}}\right) V_{\mathrm{D}}$.

c. Threshold voltage defined by linear regime MOSFET equation.

d. On-to-off current ratio at $V_{\mathrm{D}}=-0.5 \mathrm{~V}$.

e. Charge-carrier density in bulk PEDOT films extracted by using the concept of threshold voltage in OFETs.

f. Charge-carrier density in bulk PEDOT composites extracted by using the definition of conductivity. 

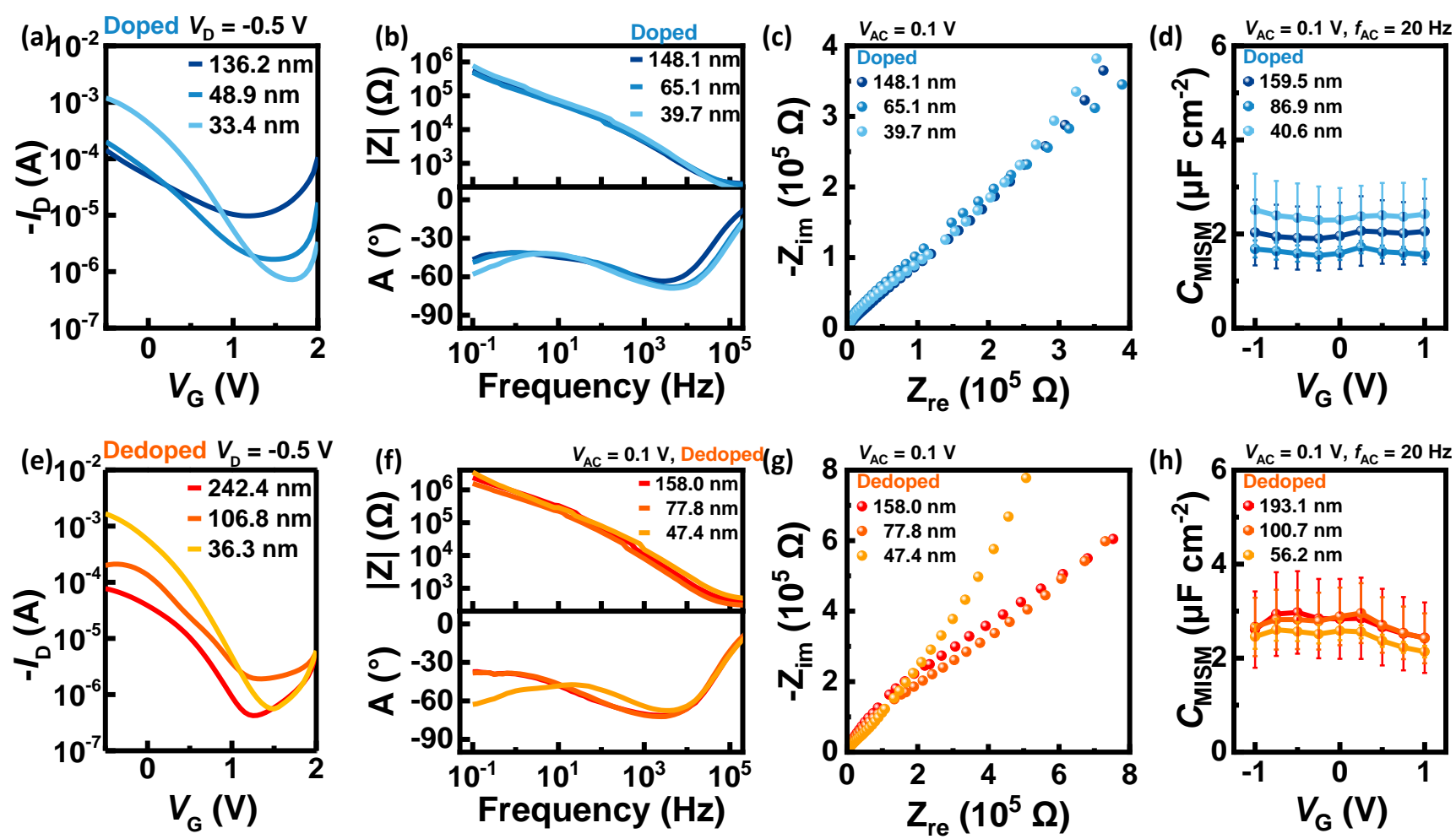

Figure S7. Thickness dependency of electrical properties of electrolyte-gated devices based on (a-d) doped and (e-h) dedoped PEDOT composites. (a,e) Transfer curves of OEGTs with a gate sweep rate of $10 \mathrm{mV} \mathrm{s}^{-1}$. (b,f) Bode plots, $(\mathrm{c}, \mathrm{g})$ Nyquist plots, and $(\mathrm{d}, \mathrm{h})$ capacitance-voltage $(\mathrm{C}-\mathrm{V})$ plots of MISM devices based on EG-PEDOT:PSS electrode/ionogel/PEDOT composite of interest with various thickness/Au. 

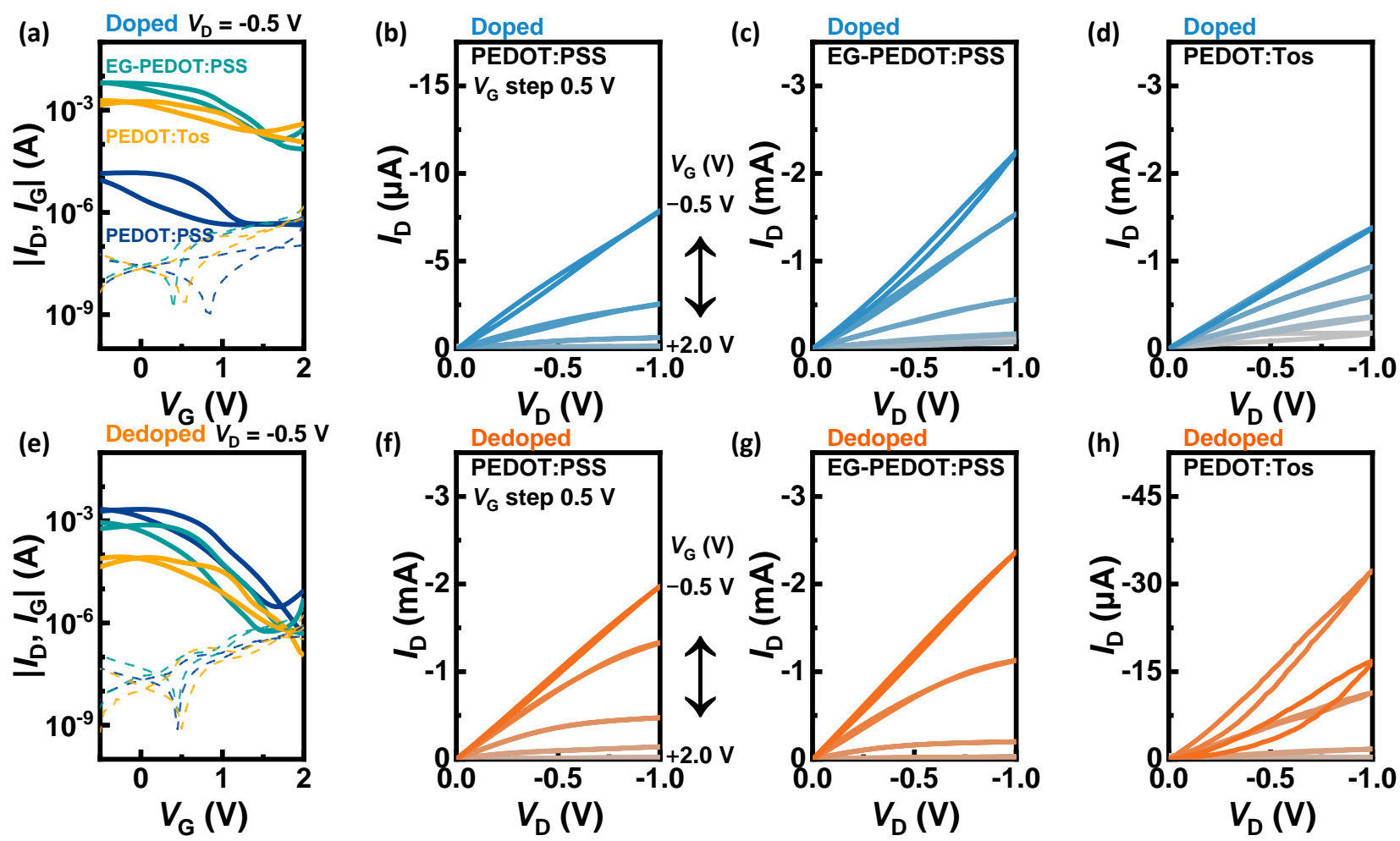

Figure S8. Current-voltage characteristics of solid-state OEGTs based on various PEDOT composites in (a-d) doped and (e-h) dedoped states. Transfer curves with gate leakage currents of (a) doped and (e) dedoped PEDOT composites. Output curves of (b-d) doped and (f-h) dedoped PEDOT composites. 

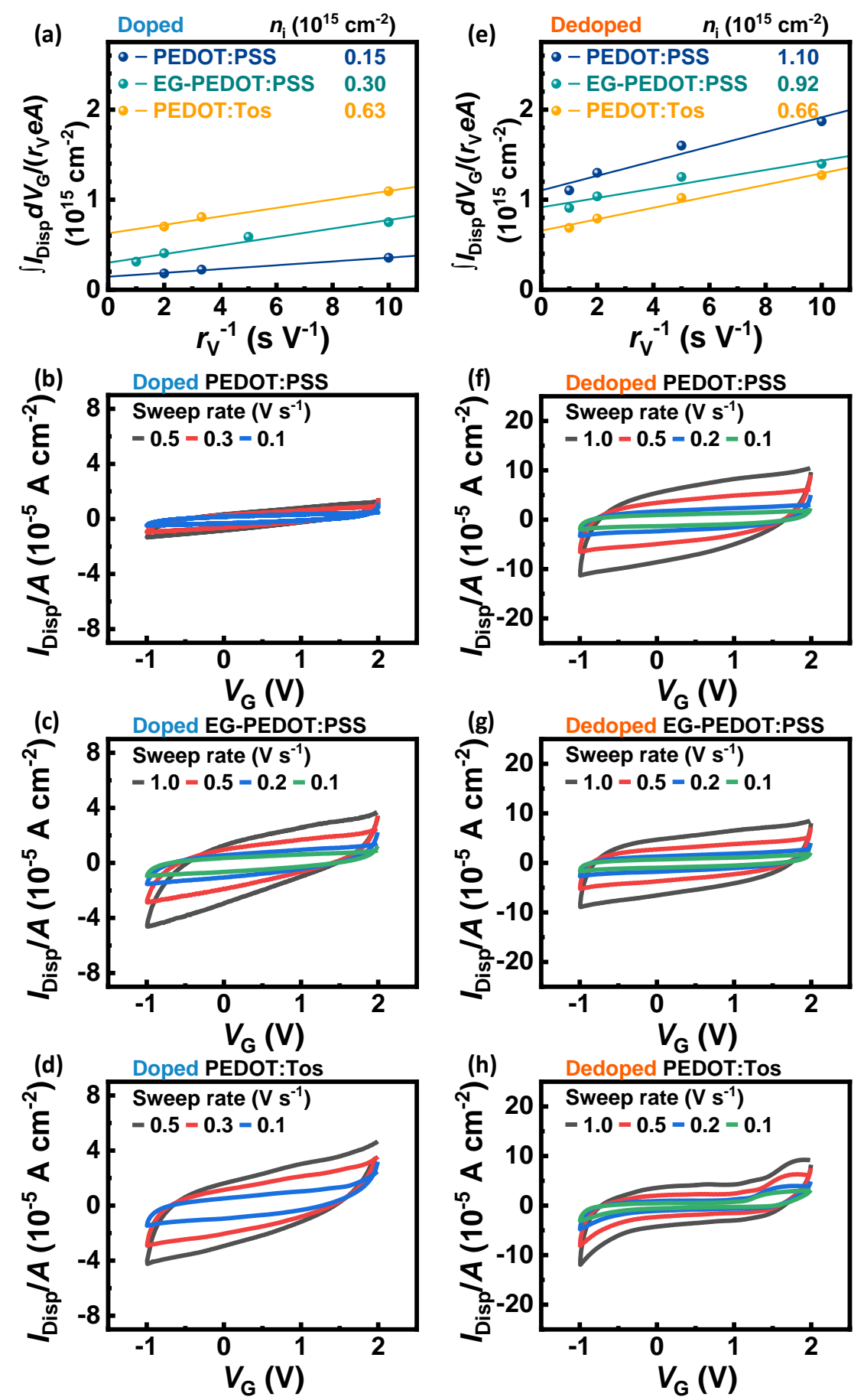

Figure S9. Displacement current behaviors of (a-d) doped and (e-h) dedoped PEDOT films. (a, e) The $n_{\mathrm{i}}=\left(\int I_{\text {Disp }} \mathrm{d} V_{\mathrm{G}}\right) /\left(e r_{\mathrm{V}} A\right)$ plot with different $r_{\mathrm{V}}{ }^{-1}$ and fitted line for $n_{\mathrm{i}}$ extraction. Dynamic displacement current measurements with various gate sweep rates from doped (b-d) and dedoped (f-h) PEDOT composite films. 
Table S4. Electrical parameters of OEGTs and MISM capacitors based on various PEDOT composites. ${ }^{\text {a }}$

\begin{tabular}{|c|c|c|c|c|c|c|}
\hline & \multicolumn{2}{|c|}{ PEDOT:PSS } & \multicolumn{2}{|c|}{ EG-PEDOT:PSS } & \multicolumn{2}{|c|}{ PEDOT:Tos } \\
\hline & Doped & Dedoped & Doped & Dedoped & Doped & Dedoped \\
\hline$V_{\mathrm{th}}^{\mathrm{b}}(\mathrm{V})$ & $\begin{array}{c}0.11 \\
\pm 0.06\end{array}$ & $\begin{array}{c}0.68 \\
\pm 0.16\end{array}$ & $\begin{array}{c}0.85 \\
\pm 0.08\end{array}$ & $\begin{array}{c}0.65 \\
\pm 0.19\end{array}$ & $\begin{array}{c}0.93 \\
\pm 0.24\end{array}$ & $\begin{array}{c}0.58 \\
\pm 0.19\end{array}$ \\
\hline $\log _{10} I_{\mathrm{ON}}$ & $\begin{array}{l}-4.8 \\
\pm 0.2\end{array}$ & $\begin{array}{l}-2.5 \\
\pm 0.1\end{array}$ & $\begin{array}{l}-2.9 \\
\pm 0.6\end{array}$ & $\begin{array}{l}-2.8 \\
\pm 0.4\end{array}$ & $\begin{array}{l}-2.8 \\
\pm 0.1\end{array}$ & $\begin{array}{l}-4.2 \\
\pm 0.4\end{array}$ \\
\hline $\log _{10} I_{\mathrm{OFF}}$ & $\begin{array}{l}-6.9 \\
\pm 0.5\end{array}$ & $\begin{array}{l}-5.9 \\
\pm 0.4\end{array}$ & $\begin{array}{l}-4.1 \\
\pm 0.3\end{array}$ & $\begin{array}{l}-5.7 \\
\pm 0.9\end{array}$ & $\begin{array}{l}-4.0 \\
\pm 0.1\end{array}$ & $\begin{array}{l}-6.8 \\
\pm 0.2\end{array}$ \\
\hline$I_{\mathrm{ON}} / I_{\mathrm{OFF}}{ }^{\mathrm{c}}$ & 240 & $3 \times 10^{3}$ & 37 & $2 \times 10^{3}$ & 12 & $7 \times 10^{2}$ \\
\hline$g_{\mathrm{m}}^{\mathrm{d}}\left(10^{-3} \mathrm{~S}\right)$ & $\begin{array}{c}0.039 \\
\pm 0.021\end{array}$ & $\begin{array}{c}3.0 \\
\pm 0.7\end{array}$ & $\begin{array}{c}3.5 \\
\pm 2.0\end{array}$ & $\begin{array}{c}2.1 \\
\pm 1.3\end{array}$ & $\begin{array}{c}1.2 \\
\pm 0.3\end{array}$ & $\begin{array}{c}0.10 \\
\pm 0.07\end{array}$ \\
\hline $\mathrm{C}_{\mathrm{MISM}^{\mathrm{e}}}\left(10^{-6} \mathrm{~F} \mathrm{~cm}^{-2}\right)$ & $\begin{array}{l}44.3 \\
\pm 8.5\end{array}$ & $\begin{array}{l}87.1 \\
\pm 9.7\end{array}$ & $\begin{array}{l}20.2 \\
\pm 1.1\end{array}$ & $\begin{array}{l}73.4 \\
\pm 7.8\end{array}$ & $\begin{array}{l}35.2 \\
\pm 3.1\end{array}$ & $\begin{array}{l}41.1 \\
\pm 4.5\end{array}$ \\
\hline$\mu_{\mathrm{MISM}^{\mathrm{f}}}\left(\mathrm{cm}^{2} \mathrm{~V}^{-1} \mathrm{~s}^{-1}\right)$ & $\begin{array}{c}0.17 \\
\pm 0.10\end{array}$ & $\begin{array}{c}6.93 \\
\pm 1.52\end{array}$ & $\begin{array}{c}34.93 \\
\pm 19.56\end{array}$ & $\begin{array}{c}5.66 \\
\pm 3.43\end{array}$ & $\begin{array}{c}7.09 \\
\pm 1.68\end{array}$ & $\begin{array}{c}0.49 \\
\pm 0.35\end{array}$ \\
\hline$I_{\mathrm{on}}^{\mathrm{g}}\left(10^{-3} \mathrm{~A}\right)$ & $\begin{array}{c}0.031 \\
\pm 0.016\end{array}$ & $\begin{array}{c}3.3 \\
\pm 1.0\end{array}$ & $\begin{array}{c}4.5 \\
\pm 2.7\end{array}$ & $\begin{array}{c}2.3 \\
\pm 1.7\end{array}$ & $\begin{array}{c}1.5 \\
\pm 0.5\end{array}$ & $\begin{array}{c}0.076 \\
\pm 0.053\end{array}$ \\
\hline$n_{\mathrm{i}}^{\mathrm{h}}\left(10^{15} \mathrm{~cm}^{-2}\right)$ & 0.15 & 1.10 & 0.30 & 0.92 & 0.63 & 0.66 \\
\hline$\mu_{\text {Disp }} \mathrm{i}^{\mathrm{i}}\left(\mathrm{cm}^{2} \mathrm{~V}^{-1} \mathrm{~s}^{-1}\right)$ & $\begin{array}{c}0.26 \\
\pm 0.13\end{array}$ & $\begin{array}{c}3.68 \\
\pm 1.14\end{array}$ & $\begin{array}{c}18.66 \\
\pm 11.25\end{array}$ & $\begin{array}{c}3.10 \\
\pm 2.29\end{array}$ & $\begin{array}{c}2.92 \\
\pm 1.09\end{array}$ & $\begin{array}{c}0.14 \\
\pm 0.10\end{array}$ \\
\hline
\end{tabular}

a. Quasi-static $I-V$ curves were measured with the gate sweep rate of $10 \mathrm{mV} \mathrm{s}^{-1}$.

b. Threshold voltage defined by linear regime MOSFET equation, $I_{\mathrm{D}}=(W / L)\left(\mu_{\mathrm{MISM}} C_{\mathrm{MISM}}\right)\left(V_{\mathrm{G}}-V_{\mathrm{th}}-0.5 V_{\mathrm{D}}\right) V_{\mathrm{D}}$.

c. On-to-off current ratio at $V_{\mathrm{D}}=-0.5 \mathrm{~V}$.

d. Maximum transconductance at $V_{\mathrm{D}}=-0.5 \mathrm{~V}$.

e. Capacitance per area from MISM capacitors with same PEDOT composite films with the $V_{\mathrm{AC}}$ of $0.1 \mathrm{~V}$, and $V_{\mathrm{DC}}$ of $V_{\mathrm{G}}$ at maximum transconductance.

f. Charge-carrier mobility extracted by linear regime MOSFET equation, $I_{\mathrm{D}}=(W / L)\left(\mu_{\mathrm{MISM}} C_{\mathrm{MISM}}\right)\left(V_{\mathrm{G}}-V_{\mathrm{th}}-0.5 V_{\mathrm{D}}\right) V_{\mathrm{D}}$.

g. On-state current at $V_{\mathrm{D}}=-0.5 \mathrm{~V}$.

h. Areal density of injected charge carriers into PEDOT composites defined by gate-displacement current measurements.

i. Charge-carrier mobility extracted by using displacement current with equation, $I_{\mathrm{D}}=(W / L)\left(\mu_{\text {Disp }} e n_{\mathrm{i}}\right) V_{\mathrm{D}}$. 

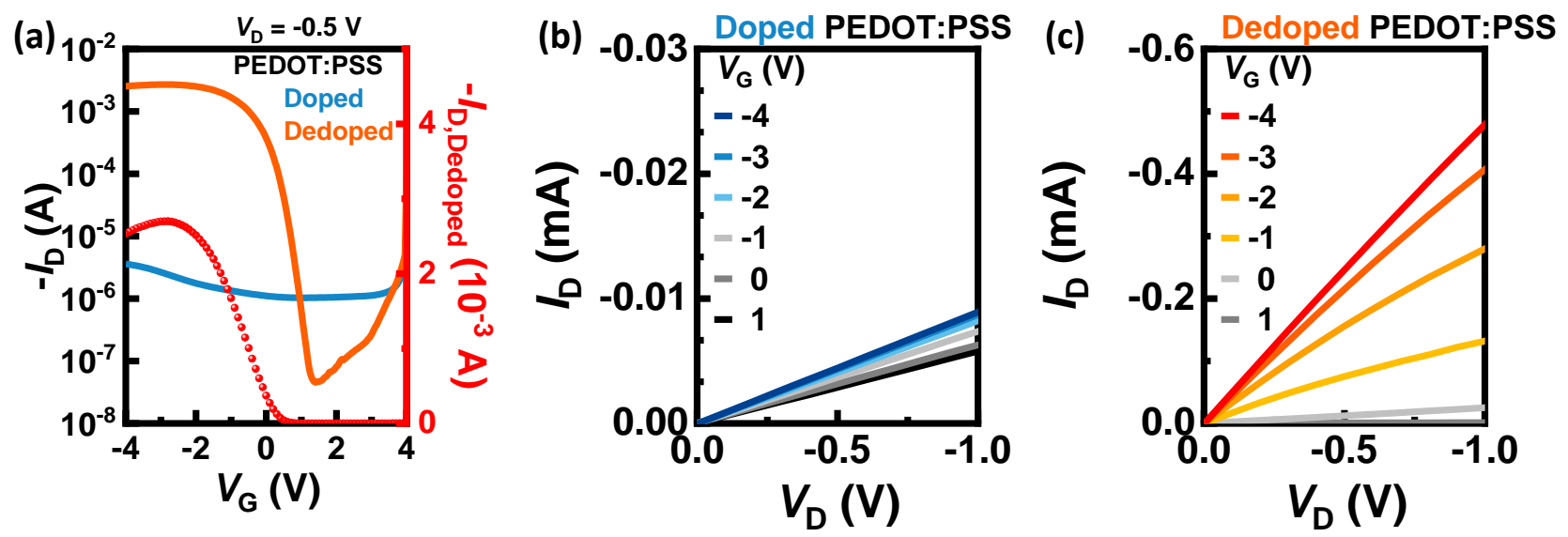

Figure S10. $I-V$ characteristics of solid-state ionogel-gated PEDOT:PSS films with the same sweep condition as dynamic UV-Vis-NIR measurement. (a) Transfer curve of doped and dedoped PEDOT:PSS films with a sweep rate of $100 \mathrm{mV} \mathrm{s}^{-1}$. Output curves of (b) doped and (c) dedoped PEDOT:PSS films as a function of gate and drain voltages. 